\title{
Molecular characterization of genome of a novel human T-cell leukaemia virus
}

\author{
Irvin S. Y. Chen*, Jami McLaughlin*, Judith C. Gasson*, Steven C. Clark ${ }^{\dagger}$ \\ \& David W. Golde*
}

* Division of Hematology-Oncology, Department of Medicine, UCLA School of Medicine, Los Angeles, California 90024, USA

$\dagger$ The Genetics Institute, 225 Longwood Avenue, Boston, Massachusetts 02115, USA

A novel human retrovirus (HTLV-II) was previously found associated with a T-cell variant of hairy-cell leukaemia. Molecular cloning demonstrates that the complete provirus genome is 8.8 kilobase pairs in size and is transmissible to uninfected cells. Two types of infectious deleted provirus were also characterized. The sequences of HTLV-II are distinct from those of HTLV-I.

HUMAN T-cell leukaemia virus is associated with human Tlymphocyte-derived malignancies in various regions of the world $^{1-3}$. Most of these virus isolates, termed HTLV-I or ATLV, are similar as shown by immunologic and molecular hybridization studies ${ }^{4}$. Recently, a new subtype of HTLV was described which was related but immunologically distinct from the more common HTLV isolates ${ }^{5}$. This virus, termed HTLV-II, was present in a cell line (Mo) established from spleen cells of a patient with a disease different from the typical HTLV-I-associated disease ${ }^{6,7}$. This patient had a relatively benign $T$-cell variant of hairy-cell leukaemia, and he is alive and well 7 years after splenectomy. To date, no other isolates of this subtype have been described. Both HTLV-I and HTLV-II are capable of transforming normal human peripheral blood cells by co-cultivation with virus-infected cells ${ }^{8-11}$.

Little is known about the relationship between the genome structure of the two subtypes of HTLV and the diseases with which they are associated. The genome structure of HTLV-I is typical of that of retroviruses ${ }^{12,13}$. The proviral DNA consists of two long terminal repeats (LTR) separated by approximately 8.0 kilobase pairs $(\mathrm{kbp})$. The genome structure of HTLV-II has not been previously characterized. We demonstrate here that the proviral genome of HTLV-II is about $8.8 \mathrm{kbp}$ in size. In addition, several viral genomes with internal deletions are characterized. Hybridization studies show that the DNA sequences of HTLV-II are distinct from those of HTLV-I.

\section{cDNA clones to HTLV-II mRNA}

Since viral-specific mRNA in retrovirus-infected cells is usually a high percentage of the total mRNA, we first isolated viral cDNA clones from mRNA of HTLV-II-infected cells. Several viral cDNA clones were isolated from a cDNA library constructed from poly(A) ${ }^{+}$RNA of Mo cells (Fig. 1). The clones were identified by 'colony hybridization' with a cDNA probe made from virion RNA.

The cDNA clones were shown to be viral specific by Southern hybridization $^{14}$ to DNA isolated from several neoplastic human cell lines (data not shown). The cDNA clones were homologous to DNA from HTLV-II-transformed cell lines (Mo and transformants derived by co-cultivation with $\mathrm{Mo})^{11}$, and not homologous to DNA from other lymphoid or myeloid cells, including transformed cell lines infected with HTLV-I, confirming that these cDNA clones are HTLV-II specific.

Since these cDNA clones were synthesized from Mo poly(A)containing RNA using oligo(dT) as a primer for the first DNA strand, most of the clones should be representative of the $3^{\prime}$ end of the viral genome. Nucleic acid sequencing of one viral cDNA ( $\mathrm{pH}-1)$ showed that this clone was terminated by a long stretch of adenylic acid residues (Fig. 1), consistent with the sequences of this clone representing the $3^{\prime}$ end of the genome. The partial sequence of this clone showed no significant homology with the previously published sequence of the $3^{\prime}$ end of ATLV (HTLV-I ${ }^{12}$. However, like ATLV, HTLV-II lacks the usual 'signal' for polyadenylation (AAUAAA), which is generally located $10-30$ bases upstream from the polyadenylation site.

Hybridization and nucleic acid sequencing of a second cDNA clone $(\mathrm{pH}-3)$ showed that it overlapped about 70 bases at the $5^{\prime}$ end of $\mathrm{pH}-1$. The viral sequences of these two cDNA clones together represent about $1.0 \mathrm{kbp}$ of the $3^{\prime}$ end of the viral genome. A third cDNA clone, $\mathrm{pH}-13$, was not homologous to either of the first two but was found to be homologous to the $5^{\prime}$ end of the viral genome (see below).

\section{Transmission of HTLV-II sequences}

Unintegrated linear viral DNA is synthesized following infection of cells by retroviruses. The viral cDNA clones were used as hybridization probes to study the structure of the unintegrated linear form of HTLV-II DNA in infected cells. It was previously shown that HTLV-II could be transmitted to normal human peripheral blood cells by co-cultivation with Mo cells ${ }^{11}$. This method was used to infect a human T-lymphoblast cell line, CEM, with HTLV-II by co-cultivation of CEM cells with several HTLV-II-infected cell lines. The unintegrated linear viral DNA was prepared from the infected cells by the extraction method of Hirt $^{15}$, and detected in infected cells by hybridization using viral cDNA clones (Fig. 2). Unintegrated linear viral DNA was detected in all co-cultures. No unintegrated viral DNA was detected in CEM cells or Mo cells alone (data not shown). Therefore, these experiments demonstrate the transmission of HTLV-II from the infected cells to the uninfected CEM cells.

An $8.8 \mathrm{kbp}$ species of viral DNA was the major form of HTLV-II DNA in the infected CEM cells. Since its size is similar to that of other replication-competent retroviruses, it is likely to represent the replication-competent HTLV-II genome. Other less prominent species of viral DNA were also detected. These may represent defective viral genomes or circular forms of viral DNA.

\section{Cloning HTLV-II proviral sequences}

The genome structure of HTLV-II was further characterized by obtaining molecular clones of proviral sequences from Mo DNA. A $\lambda$ recombinant DNA library was screened for HTLV-II sequences by hybridization with the cDNA clones. The structure of three representative proviral clones is shown in Fig. 3. 


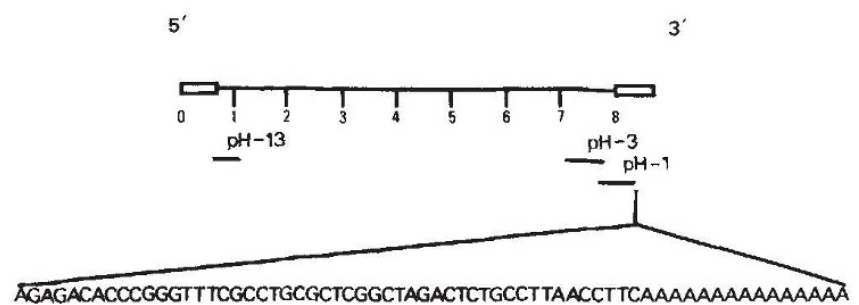

Fig. 1 Viral cDNA clones derived from Mo poly(A)-containing RNA. The cDNS clones were isolated by 'colony hybridization' from a cDNA library prepared from poly(A)-containing RNA of Mo cells ${ }^{23}$. The hybridization probe was synthesized from sucrose gradient-banded virions in a detergent-activated endogenous reaction $^{24}$. The DNA genome of HTLV-II (see Fig. 3) is shown schematically. The relative position of three viral cDNA clones is shown below the viral genome. The nucleic acid sequence of the $3^{\prime}$ end of $\mathrm{pH}-1$ was obtained by the method of Sanger $^{2}$ as modified $^{26}$ using a synthetic oligonucleotide primer (PL Biochemicals) homologous to the region immediately adjacent to one side of the PstI site of pBR322.

The DNA clone having the most proviral sequences was $\lambda \mathrm{H}-6$. Two long terminal repeats (LTRs) were identified by hybridization with $\mathrm{pH}-1$ which represents the $3^{\prime}$ end of the viral genome (see above) and therefore probably all of the U3R region ${ }^{16}$. BamHI sites are also present in both $\mathrm{pH}-1$ viral sequences and at the ends of the proviral sequences in $\lambda \mathrm{H}-6$. The orientation of the provirus was determined by hybridization to $\mathrm{pH}-3$, the sequences of which represent the $3^{\prime}$ end of the genome upstream from those of $\mathrm{pH}-1$. $\mathrm{pH}-3$ did not hybridize to the LTRs, indicating that it does not include $\mathrm{U} 3$ sequences. $\mathrm{pH}-13$ hybrid-

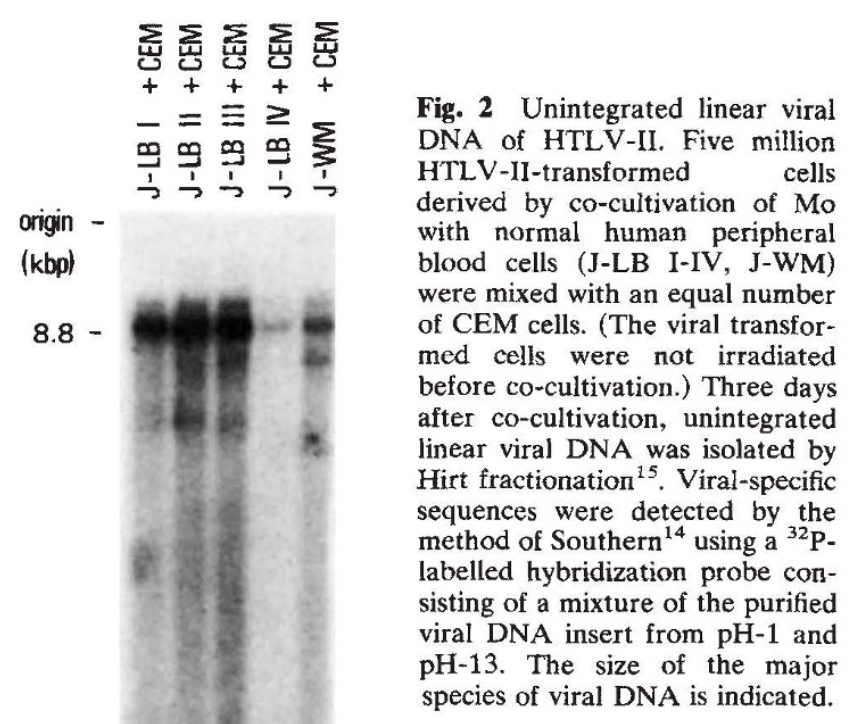

ized strongly to the $5^{\prime}$ end of the proviral genome. $\mathrm{pH}-13$ has an EcoRI site in viral sequences. $\lambda \mathrm{H}-6$ had an EcoRI site at $0.75 \mathrm{kbp}$ at the region of homology with $\mathrm{pH}-13$. pH-13 also hybridized weakly to the $3^{\prime}$ end of the genome indicating that the viral sequences in $\mathrm{pH}-13$ probably include part of the U5 region $^{16}$ of the LTR. The size of the viral sequences in $\lambda \mathrm{H}-6$ was approximately $8.8 \mathrm{kbp}$, consistent with the size of unintegrated linear viral DNA. It is likely that the provirus of $\lambda \mathrm{H}-6$ represents the complete genome of replication-competent HTLV-II. Three additional proviral clones having the same

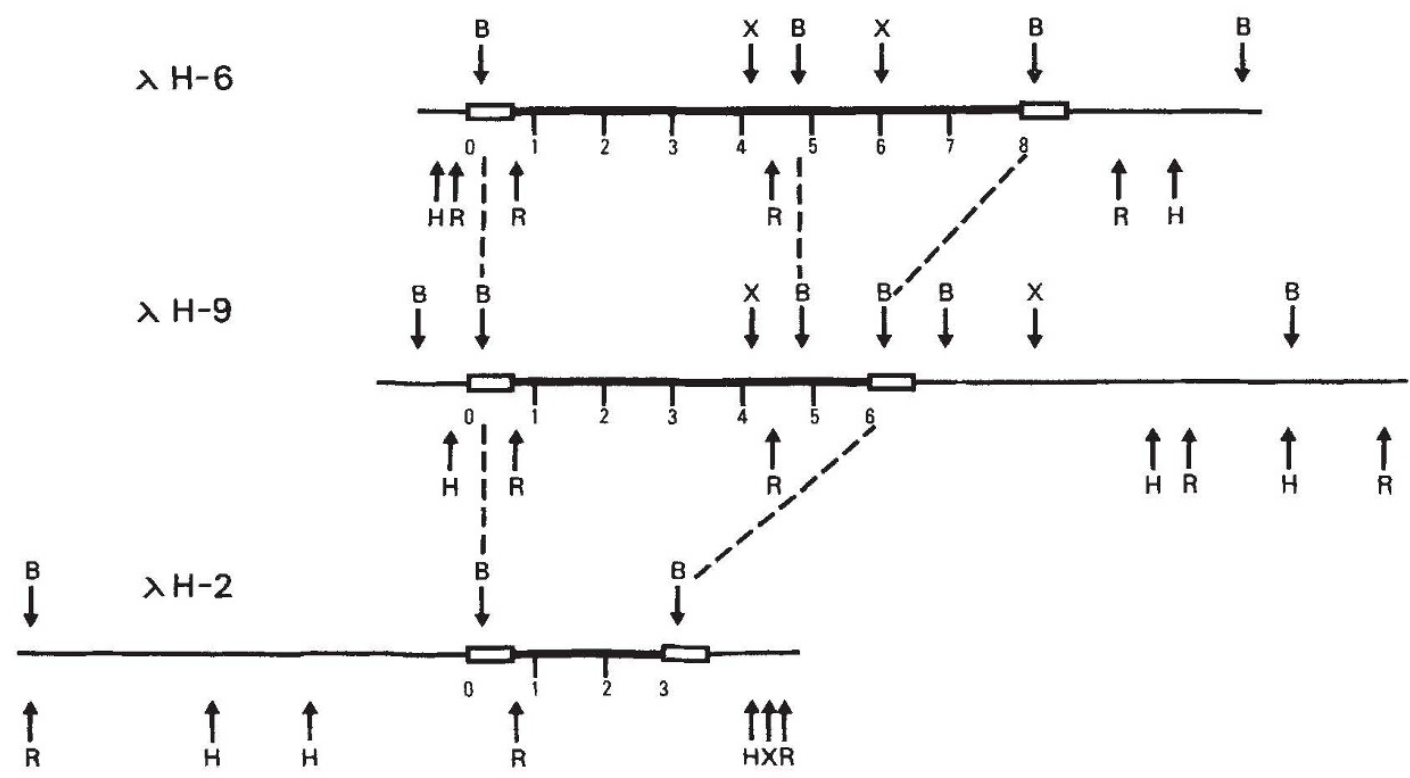

Fig. 3 Molecular clones of HTLV-II proviruses from Mo cell DNA. A $\lambda$ recombinant DNA library was constructed using $\lambda 1059^{27}$ and DNA from Mo cells. The Mo-cell DNA was digested with the restriction enzyme Sau3A for various times. The digested DNAs were pooled and fractionated by size on a $\mathrm{NaCl}$ gradient ${ }^{28}$. DNA fragments between 10 to $25 \mathrm{kbp}$ were isolated and ligated to $\lambda 1059 \mathrm{DNA}$ digested with $B a m$ HI. Recombinant phage were recovered by in vitro packaging ${ }^{29}$. The recombinant phage were screened for viral sequences by hybridization using the purified viral DNA inserts from $\mathrm{pH}-1$ and $\mathrm{pH}-13$ (see Fig. 1). The cloned inserts representing three HTLV-II proviruses are shown. The viral sequences including LTRs are represented by the dark solid bar flanked by two open boxes. The light lines represent the flanking human cellular sequences. The $5^{\prime}$ and $3^{\prime}$ ends of the viral genome are shown. The sites of cleavage for the restriction enzymes BamHI, EcoRI, HindIII, Xhol are shown. The numbers on the genomes indicate size in kbp. The corresponding BamHI sites between different viral clones are indicated by the dotted lines. 


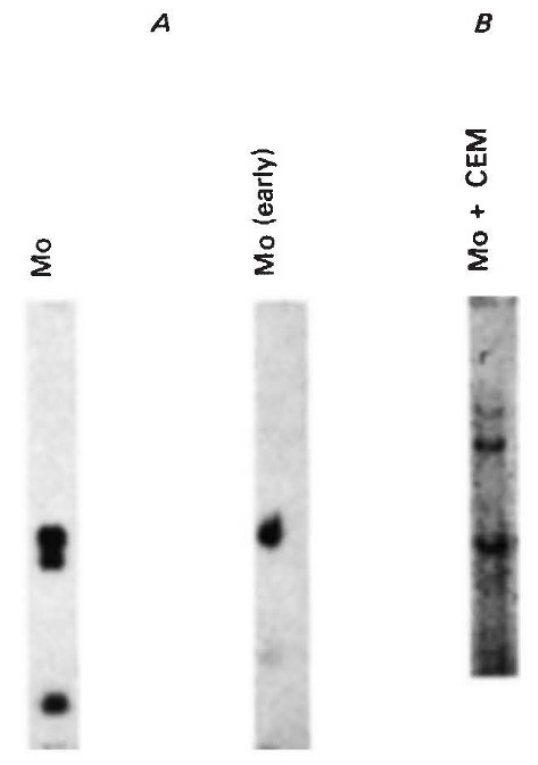

Fig. 4 Presence of defective viral genomes in Mo cells. $A, 15 \mu \mathrm{g}$ of DNA from Mo cells and an early passage of Mo cells (Mo (early)) were digested with BamHI and hybridized by the method of Southern with ${ }^{32} \mathrm{P}$-labelled $\mathrm{pH}-3$. The sizes of the DNA fragments characteristic of the three proviruses are indicated. $B$, Unintegrated linear viral DNA was prepared after co-cultivation of the later-passage Mo cells and CEM cells and viral-specific sequences were detected as described in the legend to Fig. 2. The sizes of the unintegrated linear viral DNA characteristic of the three viruses are indicated.

pattern of restriction enzyme sites and hybridization to cDNA clones in viral sequences were isolated from Mo DNA. Two of these represented the same provirus as $\lambda \mathrm{H}-6$ with different amounts of the same cellular DNA flanking the provirus. One other proviral clone had the same proviral sequences but differed from $\lambda \mathrm{H}-6$ in the cellular sequences surrounding the provirus. In addition, two other different proviruses of the same structure as $\lambda \mathrm{H}-6$ were cloned from the DNA of an HTLV-II transformant (J-LB II $)^{11}$ derived by co-cultivation with Mo cells.

The pattern of cleavage by restriction endonucleases differed between that of HTLV-II DNA and that of the previously published map of $\mathrm{ATLV}^{12}$. For example, no EcoRI sites are present in ATLV.

In addition to the apparently complete proviruses typified by $\lambda \mathrm{H}-6$, other proviruses were isolated from Mo DNA which contained large internal deletions. One of these is represented by $\lambda \mathrm{H}-9$ (Fig. 3). This provirus has a large deletion of about $2.2 \mathrm{kbp}$ between the $\mathrm{BamHI}$ sites located at 4.9 and $8.3 \mathrm{kbp}$ at the $3^{\prime}$ end of the virus. Other regions of the viral genome were not grossly altered. Three other proviral clones with the same deletion were also isolated. One of these represented the same provirus as $\lambda \mathrm{H}-9$. The other two viral clones represented proviruses with the same deletion but integrated in different cellular DNA sequences.

A third type of provirus was also identified by molecular cloning from Mo DNA. This provirus was represented by $\lambda \mathrm{H}-2$ (Fig. 3) and consisted of a viral genome with a large deletion of about $6.0 \mathrm{kbp}$ which removed most of the internal sequences of the viral genome. The LTRs appeared to be intact since both ends of the provirus hybridized with the cDNA clones $\mathrm{pH}-1$ and $\mathrm{pH}-13$. The EcoRI site at $0.75 \mathrm{kbp}$ and present in $\mathrm{pH}-13$ was conserved at the $5^{\prime}$ end of the viral genome as were the BamHI sites present in $\mathrm{pH}-1$ and the LTRs. Some sequences homologous to $\mathrm{pH}-3$ also were conserved. Two proviruses of this type were isolated which differed in integration sites.

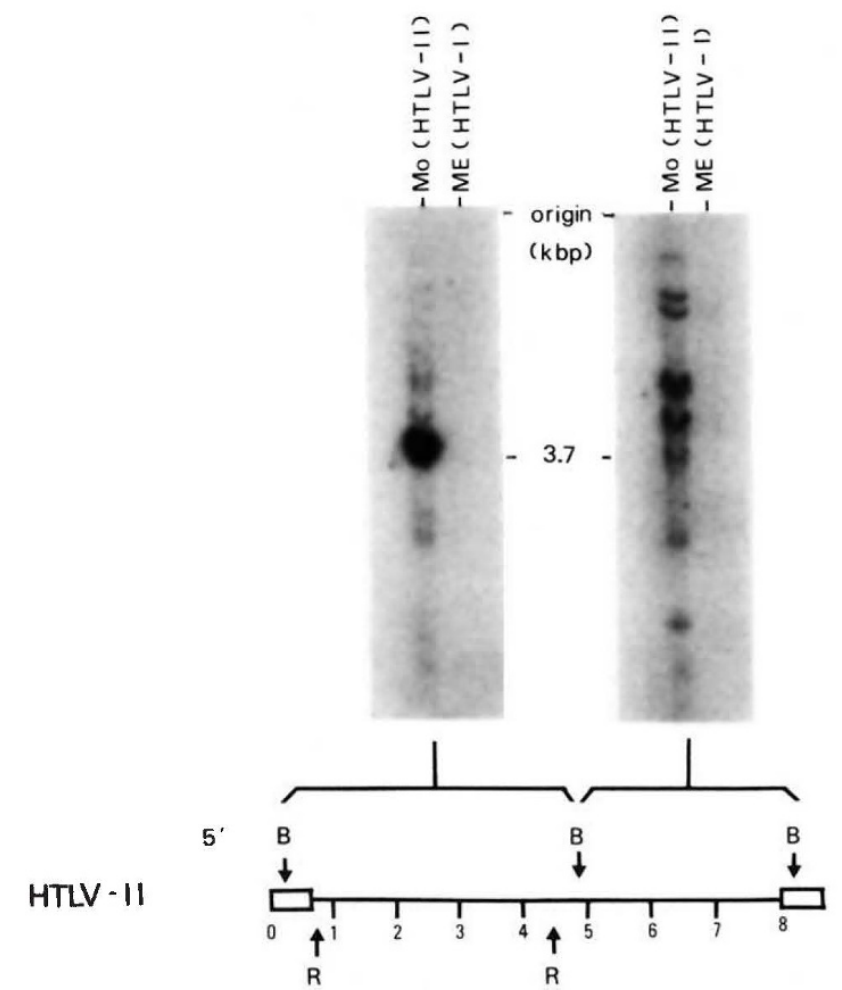

Fig. 5 Hybridization of HTLV-II sequences to Mo and ME cell DNA. Mo and ME cell DNA were digested with EcoRI, fractionated in parallel by agarose gel electrophoresis and hybridized by the method of Southern ${ }^{14}$ with probes made from different regions of HTLV-II. Each lane represents $15 \mu \mathrm{g}$ of cellular DNA. The source of the hybridization probes with respect to the HTLV-II genome is shown below the autoradiographs. The $5^{\prime}$ and $3^{\prime}$ ends of the genome are indicated and the sites of cleavage for the restriction enzymes $B a m \mathrm{HI}$ and $E c o R I$ are indicated. The numbers on the HTLV genome indicate size in kbp. The major DNA fragment of $3.7 \mathrm{kbp}$ represents the internal EcoRI fragment of the HTLV-II genome (see map). All other DNA fragments detected represent proviral sequences from the EcoRI sites in HTLV-II DNA extending $5^{\prime}$ or $3^{\prime}$ to the next proximal EcoRI site in cell DNA.

The three different HTLV-II genomes are present in Mo DNA as evidenced by analysis of Mo DNA by Southern hybridization. Clone pH-3 is homologous to the $3^{\prime}$ end of the HTLV-II genome (see Fig. 1). Cleavage of the three proviruses typified by $\lambda \mathrm{H} 6, \lambda \mathrm{H} 9$ and $\lambda \mathrm{H} 2$ (see Fig. 3) with BamHI followed by hybridization with $\mathrm{pH}-3$ should detect characteristic viral DNA fragments of $3.4 \mathrm{kbp}, 1.2 \mathrm{kbp}$ and $2.9 \mathrm{kbp}$, respectively. Figure $4 A$ (lane marked 'Mo') shows that DNA fragments of the expected sizes are present in Mo DNA. Therefore the defective forms were not generated during molecular cloning.

DNA extracted from an early passage of the Mo cells did not have the two deleted proviruses (Fig. $4 A$, lane marked 'Mo (early)'). The 3.4-kbp fragment characteristic of the complete HTLV-II genome is present. An additional fragment of $1.7 \mathrm{kbp}$ is just detectable. Whether this new fragment represents another defective genome is unknown.

The two defective forms of HTLV-II are infectious since proviruses with the same structure were molecularly cloned and found to be integrated in different human DNA sequences (see above). Also, co-cultivation of Mo cells with CEM cells (similar to the experiment described in Fig. 2 where HTLV-II transformants were used) resulted in the appearance of three species of unintegrated linear viral DNA, the sizes of which are consistent with the sizes of the three molecularly cloned proviruses (8.8, 6.5 and $3.5 \mathrm{kbp}$ ) (Fig. $4 B$ ). 


\section{HTLV-II and HTLV-I genomes}

The pattern of restriction endonuclease cleavage sites for HTLV-II (Fig. 3) was different from that published for HTLV-I, and the sequence of the $3^{\prime}$ end of HTLV-II mRNA (Fig. 1) showed no similarities to the $3^{\prime}$ end of HTLV-I mRNA. Further comparison of the HTLV-II and HTLV-I genome was made by subcloning regions of the HTLV-II genome to use as hybridization probes to DNA from HTLV-I-infected cells.

An HTLV-I-infected cell line, ME, was derived from a patient with typical HTLV-I-associated T-cell leukaemia ${ }^{17}$. This cell line was not directly tested for HTLV-I viral sequences; however, the ME cells express viral p19 and p24 antigens as detected by immunofluorescence; the supernatant medium from the cells has sedimentable reverse transcriptase activity, and the $\mathrm{ME}$ cells can be used in co-cultivation experiments to transform normal human peripheral blood lymphocytes ${ }^{17,18}$.

Two subclones which together comprise nonoverlapping regions of the entire HTLV-II genome were constructed. The subclones consisted of viral sequences of $\lambda \mathrm{H}-6$ from the $B a m H I$ site in the $5^{\prime}$ LTR to the BamHI site at $4.9 \mathrm{kbp}$ and from this latter BamHI site to the BamHI site in the 3' LTR. In standard conditions of hybridization both subclones hybridized strongly to Mo DNA (Fig. 5). The internal 3.7-kbp EcoRI fragment was detected by the $5^{\prime}$ BamHI probe. All other bands detected by both the $5^{\prime}$ and $3^{\prime}$ probes represent proviral sequences plus adjacent cellular sequences. The large number of these fragments indicate multiple proviruses in Mo DNA. Neither of these probes, which together represent the entire HTLV-II genome, hybridized to ME DNA in the same standard conditions of hybridization. Therefore, the two subtypes of HTLV appear to be only distantly related.

\section{Conclusions}

The structure of the HTLV-II provirus is consistent with the structures of other replication-competent retroviruses. Although HTLV-II appears to be capable of rapid transformation in vitro ${ }^{11}$, no oncogenes related to normal cellular sequences have been detected in the viral genome (unpublished observation).

The genome of HTLV-II is similar in size $(8.8 \mathrm{kbp})$ to that of HTLV-I. Also, the viral genomes are similar at the $3^{\prime}$ end in that the usual signal for polyadenylation (AAUAAA) is not present; however, the nucleic acid sequences of the genomes of the two subtypes differ considerably. The pattern of restriction enzyme cleavage sites is distinct and nucleic acid hybridization shows that the viral genomes are not homologous.

The differences in nucleic acid sequence between the two subtypes of HTLV may relate to differences in the disease phenotypes associated with the viruses. HTLV-II was found associated with a single case of a relatively benign $T$-cell variant of hairy-cell leukaemia ${ }^{6,7}$, whereas. HTLV-I is usually found

Received 13 June; accepted 9 August 1983

1. Poiesz, B. J, et al Proc, natn. Acad Sci. U.S.A. 77, 7415-7419 (1980).

2. Hinuma $Y$ et al Proc natn. Acad Sci U.S. A 78, 6476-6480 (1981)

3. Blattner, W. A. et al. Int. J. Cancer 30, 257-264 (1982).

4. Popovic, M. et al. Nature 300,63-66 (1982).

5. Kalyanaraman, V. S. et al. Science 218, 571-573 (1982).

6. Saxon, A., Stevens, R. H., Quan, S. G. \& Golde, D. W. J. Immun. 120, 777-782 (1978)

7. Saxon, A., Stevens, R. H. \& Golde, D. W. Ann. intern. Med. 88, 323-326 (1978).

8. Miyoshi, I. et al. Nature 294, 770-771 (1981).

9amamoto, N., Okada, M., Koyanagi, Y., Kannagi, M. \& Hinuma, Y. Science 217, 737-739 (1982).

10. Popovic, M. et al. Science 219, 856-859 (1983)

11. Chen, I. S. Y., Quan, S. G. \& Golde, D. W. Proc. natn. Acad. Sci, U.S.A. (in the press).

12. Seiki, M., Hattori, S. \& Yoshida, M. Proc. natn. Acad. Sci. U.S.A. 79, 6899-6902 (1982)

13. Manzari, V, et al. Proc. natn. Acad. Sci. U.S.A. 80, 1574-1578 (1983).

14. Southern, E. M. J. molec. Biol. 98, 503-517 (1975).

15. Hirt, B. J. molec. Biol. 26, 365-369 (1967)

16. Weiss, R., Teich, N., Varmus, H. \& Coffin, J. (eds) RNA Tumor Viruses (Cold Spring Harbor Laboratory, New York, 1982). associated with highly malignant T-cell leukaemia and lymphoma $^{1-3}$. The role of HTLV-Il in human malignancy is unclear, however, since only one patient has been found to have this virus.

Given the differences in sequence between the two virus subtypes it is noteworthy that both viruses appear to have similar biological properties in vitro. Co-cultivation of normal human peripheral blood cells with either HTLV-I- or HTLV-II-transformed cells results in the transformation of the normal cells as evidenced by their continued proliferation. The properties of these transformed cells are generally similar with respect to morphology, surface phenotype, growth and the ability to transform normal cells by co-cultivation ${ }^{8-11,17,18}$. It is likely that similar properties of the viruses are responsible for their biological effects. In this regard, although their nucleic acid sequences are distinct, the viruses are partially related immunologically ${ }^{5}$. Two highly divergent retroviruses which have similar biological effects are two distinct species of avian retroviruses; avian leukaemia virus and reticuloendotheliosis virus. These viruses are both capable of causing bursal lymphomas in chickens. In this case, the viruses have common effects because of their integration next to specific oncogene sequences $(c-m y c)$ in tumour cells ${ }^{19-21}$.

The presence of infectious, defective viral genomes in HTLVinfected cells has not previously been reported. Defective viruses are common among retroviruses from other species ${ }^{16}$. Such viruses are propagated as long as there are helper viruses to provide in trans the required functions. Analysis of DNA from early passages of the Mo cells by Southern hybridization did not detect the two defective forms, although they may have been present in a small percentage of cells. In later passages of the Mo cells the defective genomes are present. Therefore, the defective forms of virus were probably generated during the passage of the cells rather than being present in the original tumour cells of the patient. A distinguishing characteristic between early and later passages of the Mo cell line is the ability of the cell line in later passages to grow serum free and to spontaneously form colonies in methylcellulose. The defective genomes in later passages of the Mo cells may be causally related to these properties. Deletions and rearrangements in transforming retroviruses of other species have been shown to modify the transforming potential of the viruses ${ }^{22}$. Deleted viruses with increased ability to transform cells can be selected for during passage of the virus ${ }^{22}$

We thank $\mathrm{K}$. Wilhelmsen for $\lambda$ in vitro packaging reagents, R. Gallo for antibodies to viral p19 and p24 antigens, S. Quan and S. Gerber for technical assistance and A. Berk, C. Westbrook and $\mathrm{O}$. Witte for helpful comments on the manuscript. This work was supported by PHS grants CA 30388 and CA 32737 awarded by the NCI, DHSS, and grant PF 2182 from the ACS.

Note added in proof: DNA from the HTLV-I-infected HUT-102 cell line did not hybridize with the $3^{\prime}$ Bam HTLV-II fragment in standard conditions.

17. Koeffler, H. P., Chen, I. S. Y. \& Golde, D. W, Blood (submitted),

18. Gasson, J. C., Chen, I. S. Y., Westbrook, C. A. \& Golde, D. W. in Normal and Neoplastic Hematopoiesis (eds Golde, D. W. \& Marks, P. A.) (Liss, New York, in the press).

19. Noori-Daloii, M. R., Swift, R. A., Kung, H.-J., Crittenden, L. B. \& Witter, R. L. Nature 294, 574-576 (1981)

20. Neel, B. G., Hayward, W. S., Robinson, H. L., Fang, J. \& Astrin, S. M. Cell 23, 323-334 (1981).

21. Payne, G. S., Bishop, J. M. \& Varmus, H. E. Nature 295, 209-215 (1982).

22. Chen, I. S. Y. \& Temin, H. M. Cell 31, 111-120 (1982).

23. Grunstein, M. \& Hogness, D. W. Proc. natn. Acad. Sci. U.S.A. 72, 3961-3965 (1975).

24. Yoshida, M., Miyoshi, I \& Hinuma, Y. Proc natm. Acad Sci. U.S.A. 79, 2031-2035 (1982)

25. Sanger, F., Nicklen, S. \& Coulson, A. R. Proc. natn. Acad. Sci. U.S.A. 74, 5463-5467 (1977).

26. Wallace, R. B. et al. Gene 16, 21-26 (1981).

27. Karn, J., Brenner, S., Barnett, L. \& Cesareni, G. Proc. natn. Acad. Sci. U.S.A. 77, $5172-5176(1980)$.

28. O'Rear, J. J., Mizutani, S., Hoffman, G., Fiandt, M. \& Temin, H. M. Cell 20, 423-430 (1980).

29. Becker, A. \& Gold, M. Proc. natn. Acad. Sci. U.S.A. 72, 581-585 (1975). 